\title{
Validation of the High Performance Conduction-Cooled Prototype LTS Pulse Coil for UPS-SMES
}

\author{
Toshiyuki Mito, Akifumi Kawagoe, Hirotaka Chikaraishi, Ryuji Maekawa, Kagao Okumura, Ryo Abe, \\ Tomosumi Baba, Tsutomu Hemmi, Masataka Iwakuma, Mitsuhiro Yokota, Hideki Ogawa, Yoshitaka Morita, \\ Kenji Yamauchi, Atsuko Kuge, and Fumio Sumiyoshi
}

\begin{abstract}
A conduction-cooled low temperature superconducting (LTS) pulse coil has been developed as a key technology for UPS-SMES. We have been developing a 1 MW, 1 s UPS-SMES for a protection from a momentary voltage drop and an instant power failure. A conduction-cooled LTS pulse coil has excellent characteristics, which are adequate for a short-time uninterruptible power supply (UPS). The LTS coil has better cost performance over the HTS coil at present and the conduction cooling has higher reliability and easier operation than the conventional cooling schemes such as pool boiling with liquid helium or forced flow of supercritical helium. To demonstrate the high performances of the LTS pulse coil, we have fabricated a prototype coil with stored energy of $100 \mathrm{~kJ}$ and have conducted cooling and excitation tests. The successful performance test results including current shut-off test with a time constant of $1.3 \mathrm{~s}$ and repeated excitation of a triangular waveform with high ramp rate are reported.
\end{abstract}

Index Terms-Conduction cooled, momentary voltage drop, superconducting pulse coil, UPS-SMES.

\section{INTRODUCTION}

$\mathbf{U}$ PS-SMES as a protection from momentary voltage drop and power failure are required for industrial fabrication facilities such as a semiconductor chip production equipment or large-sized experimental facilities for big science such as a nuclear fusion experimental device [1]-[3]. A five-year project to develop UPS-SMES is being started from 2002 fiscal year as one of the research promotion programs of the New Energy and industrial technology Development Organization (NEDO). We have developed a $100 \mathrm{~kJ}$ class UPS-SMES in order to do a principle actual proof [4]. According to the successful results of

Manuscript received September 20, 2005. This work was supported by NIFS (NIFS05ULAA102), the grand of NEDO and the grand-in aid for Scientific Research (\#16206028).

T. Mito, H. Chikaraishi, R. Maekawa, T. Baba, M. Yokota, H. Ogawa, Y. Morita, and K. Yamauchi are with the National Institute for Fusion Science, Toki, Gifu 509-5292, Japan (e-mail: mito@ nifs.ac.jp).

A. Kawagoe and F. Sumiyoshi are with Kagoshima University, Kagoshima 890-0065, Japan.

K. Okumura and A. Kuge are with Technova Inc., Chiyoda-ku, Tokyo 1000011, Japan.

R. Abe is with Shibuya Kogyo Co., Ltd., Mameda-honmachi, Kanazawa 9208681, Japan.

T. Hemmi is with the Graduate University for Advanced Studies, Toki, Gifu 509-5292, Japan.

M. Iwakuma is with Kyushu Univiersity, Higashi-ku, Fukuoka 812-8581, Japan.

Digital Object Identifier 10.1109/TASC.2006.870791

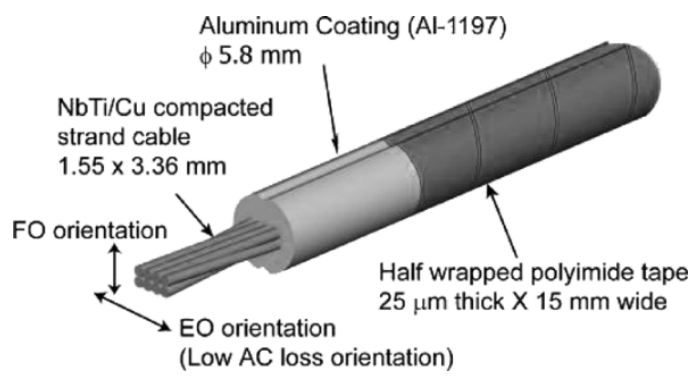

Fig. 1. Superconducting conductor for the LTS pulse coil.

TABLE I

SPECIFICATIONS OF THE SC CONDUCTOR

\begin{tabular}{|c|c|}
\hline Conductor type & $\begin{array}{l}\text { Aluminum coated } \mathrm{NbTi} / \mathrm{Cu} \text { compacted } \\
\text { strand cable }\end{array}$ \\
\hline Conductor diameter & $5.8 \mathrm{~mm}$ \\
\hline Operational current & $1000 \mathrm{~A}$ \\
\hline Critical current & $3740 \mathrm{~A} @ 5 \mathrm{~T}, 4.2 \mathrm{~K}$ \\
\hline Coupling time constant of $\mathrm{AC}$ & $82 \mathrm{msec}$ for face-on (FO) orientation \\
\hline loss: $\mathrm{A}^{*} \tau^{1)}$ & $10 \mathrm{msec}$ for edge-on (EO) orientation \\
\hline Compacted strand cable & $1.55 \times 3.36 \mathrm{~mm}$ \\
\hline Aluminum coating & Al- 1197 \\
\hline RRR of Al coating & 9.85 \\
\hline
\end{tabular}

the cooling and excitation tests, we have proceeded to construct 1 MJ LTS pulse coils used for 1 MW, $1 \mathrm{~s}$ UPS-SMES.

\section{Design And Construction of a Conduction-Cooled PROTOTYPE LTS PULSE COIL}

A low AC loss and a high stability are required for the superconducting (SC) conductor of the conduction-cooled LTS pulse coil. The SC conductor of a $\mathrm{NbTi} / \mathrm{Cu}$ compacted strand cable extruded with an aluminum has been developed as shown in Fig. 1 and its parameters are listed in Table I. The conductor has the anisotropic $\mathrm{AC}$ loss properties depending on the orientation of the time varying magnetic field. The AC loss becomes the minimum when the magnetic field is applied parallel to the flat surface of compacted strand cable (EO orientation) in the conductor. Therefore, the AC loss of the coil can be minimized as twisting the conductor during the winding process so that the EO orientation of the conductor coincides with the orientation of the magnetic field in the coil [5]. 
TABLE II

SPECIFICATIONS OF THE PROTOTYPE LTS PULSE COIL

\begin{tabular}{ll}
\hline \hline Dimension of the coil windings & \\
$\quad$ Inner diameter: $2 \mathrm{a} 1$ & $0.305 \mathrm{~m}$ \\
$\quad$ Outer diameter: $2 \mathrm{a} 2$ & $0.509 \mathrm{~m}$ \\
$\quad$ Length: $2 \mathrm{~L}$ & $0.402 \mathrm{~m}$ \\
Total turn number & 67 turns x 14 layers = 938 turns \\
Coil inductance & $0.20 \mathrm{H}$ \\
Maximum magnetic field & $2.2 \mathrm{~T}$ \\
Magnetic stored energy & $100 \mathrm{~kJ}$ \\
Operating current & $1000 \mathrm{~A}$ \\
Coil weight & $400 \mathrm{~kg}$ \\
\hline \hline
\end{tabular}

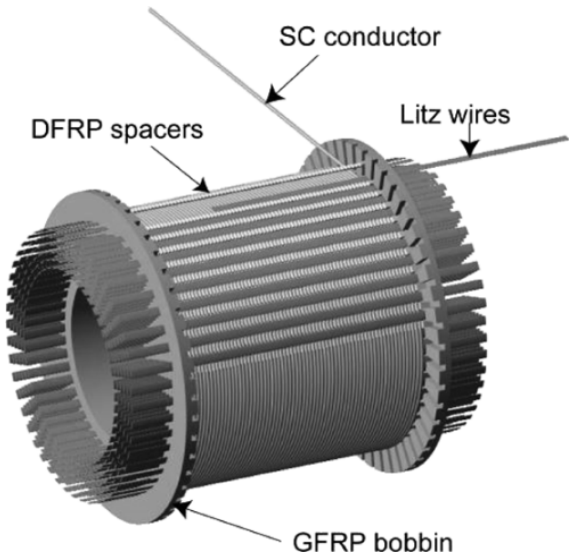

Fig. 2. Structure of conduction-cooled LTS pulse coil.

The coil shape is a single solenoid of 67 turns $\times 14$ layers wound on the GFRP bobbin using the specially developed automatic twist winding machine and its parameters are listed in Table II. The Dyneema FRP (DFRP) spacers and the Litz wires (braided wires of insulated copper strands) are inserted in each layer as shown in Fig. 2. The DFRP spacers have a good thermal conductivity along with Dyneema filaments, which enhance the heat transfer from layer to layer in the windings. On the other hand, the Litz wires increase the heat transfer from turn to turn in the windings and enable conduction cooling of the coil by attaching the end of the Litz wires directly to the cold heads of the cryocoolers. The total copper cross-section of the Litz wire is $4,774 \mathrm{~mm}^{2}$.

\section{CoOling And Excitation Tests}

\section{A. Experimental Set-Up}

Fig. 3 shows the inside of cryostat for performance tests. A conduction-cooled LTS pulse coil was connected with two GM cryocoolers which have cooling capacity of $3 \mathrm{~W}$ at $4 \mathrm{~K}$ and $120 \mathrm{~W}$ at $50 \mathrm{~K}$. Temperature sensors were placed as shown in Fig. 4 within the coil windings, thermal radiation shields and the cold heads to monitor the temperatures during cooldown and excitation tests. In addition, the heaters were attached at the inner surface for the thermal characterizations. The coil was placed in the cryostat and evacuated for the performance test.

\section{B. Cooling Test}

Two different cooldown schemes were conducted to investigate thermal performance of the coil; a precooling with a liquid nitrogen and without it. As utilizing a $\mathrm{LN}_{2}$, the cooldown of

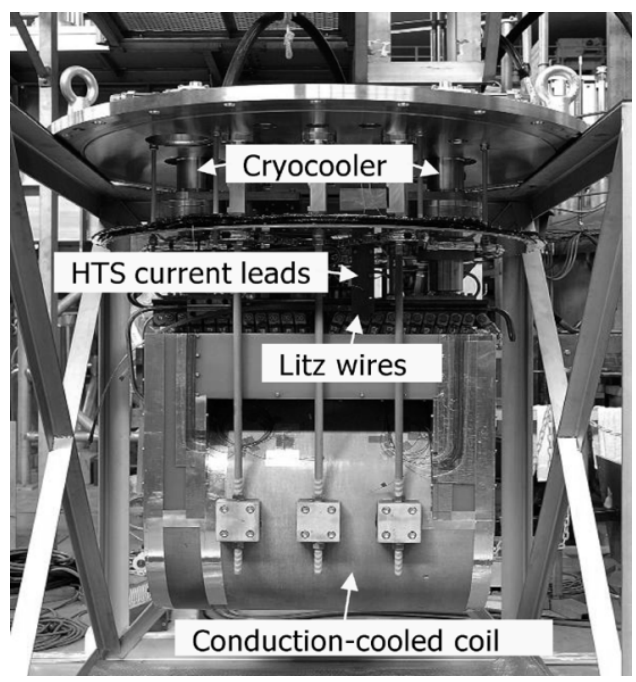

Fig. 3. Cooling and excitation test set-up of conduction-cooled LTS pulse coil.

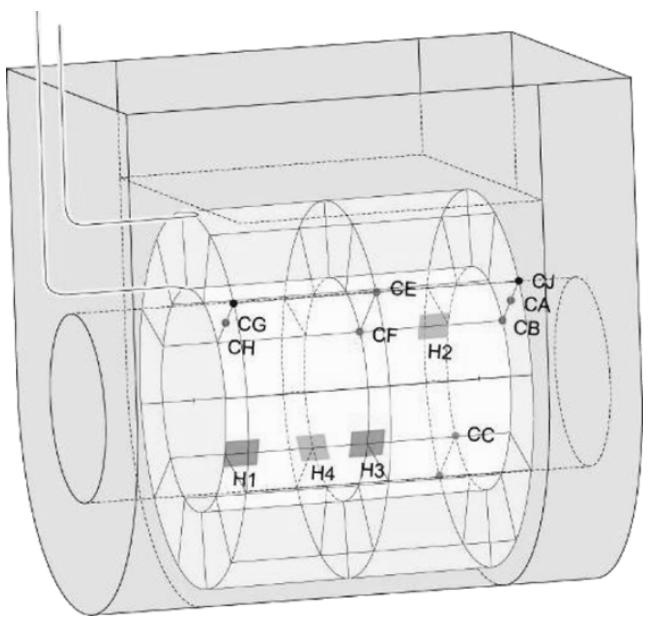

Fig. 4. Temperature sensors (CA-CJ) and heaters (H1-H4) within the coil.

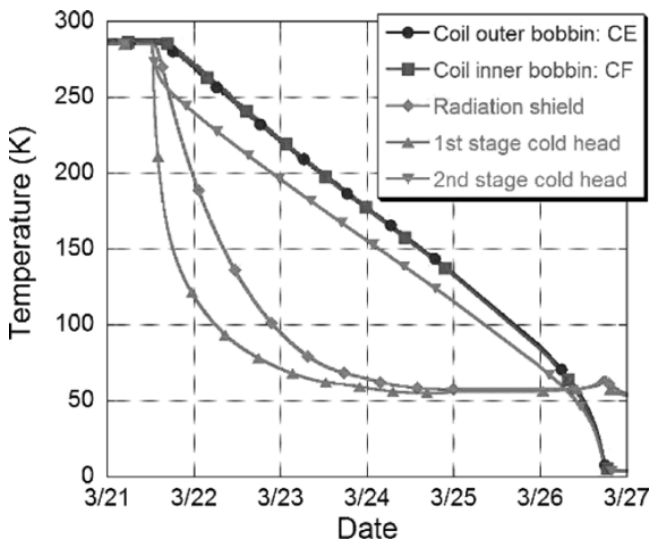

Fig. 5. Cooldown curve of conduction-cooled coil with cryocoolers.

the system was achieved within three days of operation, while it took approximately five days to cooldown the system as exclusively utilizing cryocoolers (See Fig. 5). In both cases, the spatial temperature distributions within the coil were negligible and the coil temperatures reached below $4 \mathrm{~K}$, which demonstrated the good thermal performance of the coil. 

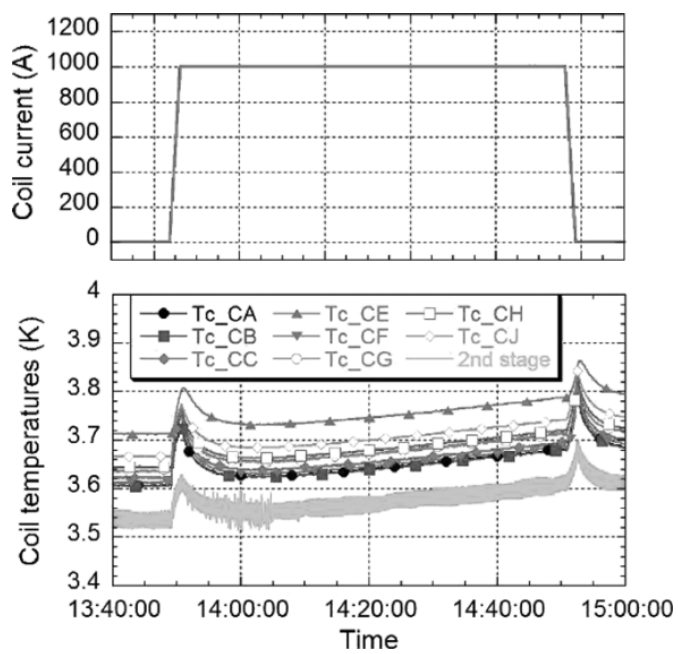

Fig. 6. Performance test results applying a rated current of $1000 \mathrm{~A}$ for an hour.
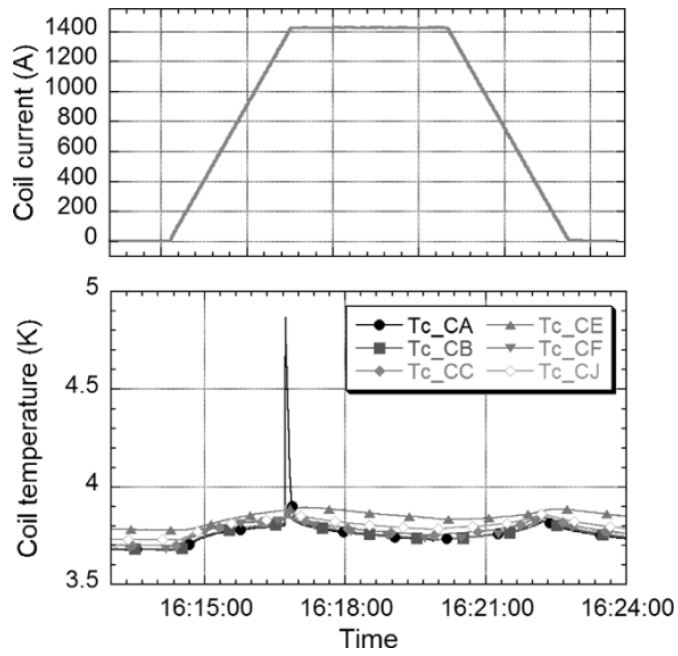

Fig. 7. Over-current test of $1420 \mathrm{~A}$ with a stored energy of $200 \mathrm{~kJ}$.

\section{Rated Current Test}

The excitation test was performed as the system reached the steady-state condition. Fig. 6 shows the performance test results. As applying a rated standby current of $1000 \mathrm{~A}$ for an hour, the temperatures within the coil was gradually increased. The slopes of temperature curves were almost identical within the coil winding, which also corresponded to the temperature increase at the cold head of cryocoolers. The results indicated that the design of a coil was proved to be sufficient to sustain stable operation with a rated current during a standby state.

\section{Over-Current Test}

Since the prototype showed a very stable operation under a rated current of $1000 \mathrm{~A}$, the test was continued to examine the maximum operating current. Fig. 7 shows the performance test with the maximum current, $1420 \mathrm{~A}$ and a stored energy of 200 $\mathrm{kJ}$. One of the temperature sensors measured a sharp temperature increase up to $4.9 \mathrm{~K}$ corresponding to a crack of epoxy resign due to the large electromagnetic force during ramp up to 1420 A. However there was no sign of the coil quench. The coil

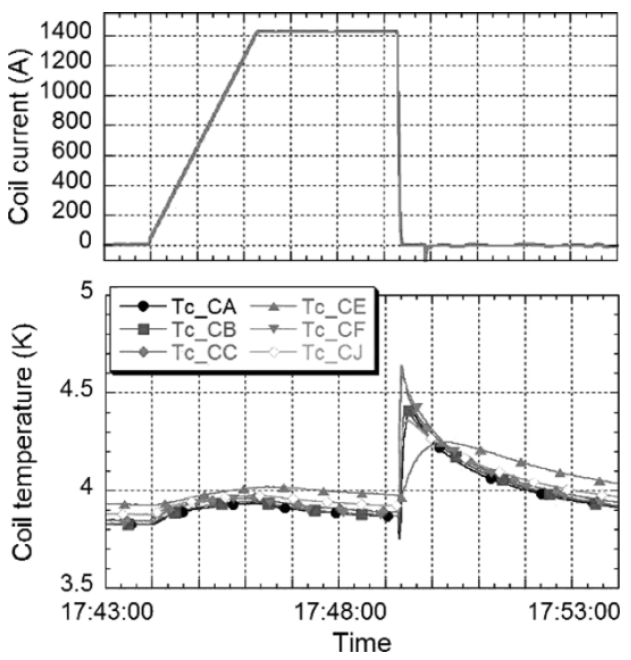

Fig. 8. High speed current shut-off test from 1420 A with time const. of $1.3 \mathrm{~s}$.

has been excited up to 1420 A repeatedly and was stably operated for one hour. The prototype proved its high potentiality of operation.

\section{E. High Speed Shut-Off Test}

To validate the thermal performance of the coil, the coil current was reduced rapidly from $1000 \mathrm{~A}, 1230 \mathrm{~A}$ and $1420 \mathrm{~A}$ to $0 \mathrm{~A}$ with a time constant of $1.3 \mathrm{~s}$. Fig. 8 shows the temperature increase in the coil during the discharge from $1420 \mathrm{~A}$. The $\mathrm{AC}$ losses of discharges from $1000 \mathrm{~A}, 1230 \mathrm{~A}$ and $1420 \mathrm{~A}$ are $147 \mathrm{~J}$, $210 \mathrm{~J}$ and $278 \mathrm{~J}$, respectively. The temperature increase within the coil was less than $0.8 \mathrm{~K}$ so that the heat generated by the AC loss was well distributed within the coil winding. Since the actual system is operated with $1 \mathrm{~s}$ discharge mode from 1000 A to $707 \mathrm{~A}$ (half of the magnetic stored energy), the test results exceed the expected performance of the coil.

\section{F. Repeated High Speed Excitation Test}

A high thermal diffusivity of the coil was demonstrated with a repeated excitation test. The current was repeatedly increased or reduced from $0 \mathrm{~A}$ to $1000 \mathrm{~A}$ with a ramp rate of $35 \mathrm{~A} / \mathrm{s}, 50$ $\mathrm{A} / \mathrm{s}$ and $75 \mathrm{~A} / \mathrm{s}$. Fig. 9 shows that the total of 20 excitations of 50 $\mathrm{A} / \mathrm{s}$ was conducted with $1.1 \mathrm{~K}$ of the coil temperature increase. The AC loss powers of the repeated high speed excitation up to $1000 \mathrm{~A}$ with the ramp rate of $35 \mathrm{~A} / \mathrm{s}, 50 \mathrm{~A} / \mathrm{s}$ and $75 \mathrm{~A} / \mathrm{s}$ were $1.2 \mathrm{~W}, 1.9 \mathrm{~W}$ and $2.9 \mathrm{~W}$, respectively. Whereas the measured temperature increases in the coil were $0.7 \mathrm{~K}, 1.1 \mathrm{~K}$ and $1.4 \mathrm{~K}$, which reveals a good thermal diffusivity of coil winding. Consequently, the coil has a potential to perform stable condition even under continuous pulse operations.

\section{DISCUSSION}

The design of conduction-cooled pulse coil is determined by the temperature margin during pulse operation. The temperature increase of the prototype coil after $1 \mathrm{~s}$ discharge is estimated as $6.7 \mathrm{~K}$ assuming an adiabatic condition in which the heat transfer from the SC conductor to the DFRP spacers and other winding components was neglected. According to the performance test, the prototype demonstrated its high potentiality for 


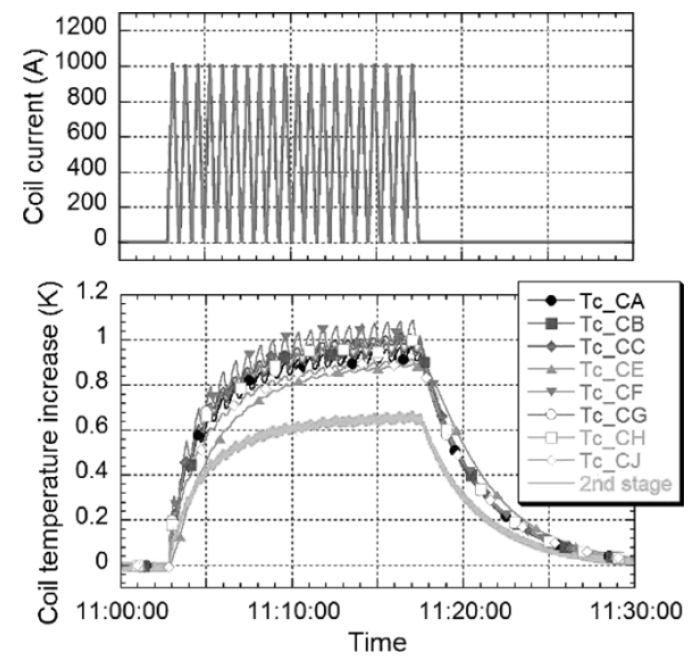

Fig. 9. 20 times repeated high speed excitations test increased or reduced from $0 \mathrm{~A}$ to $1000 \mathrm{~A}$ with a ramp rate of $50 \mathrm{~A} / \mathrm{s}$.

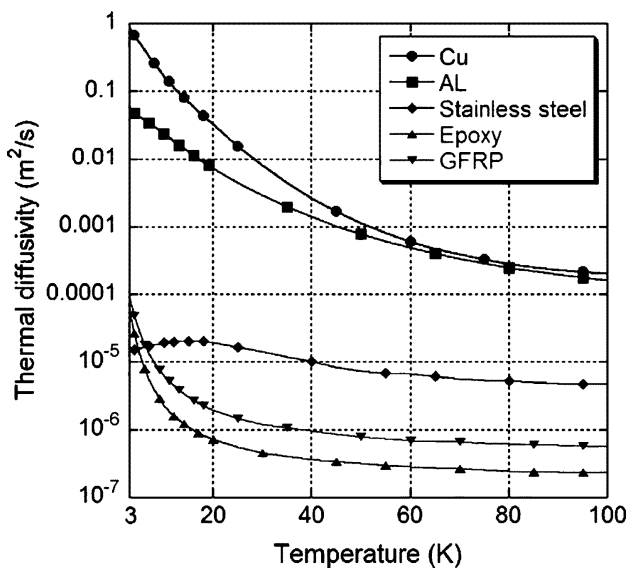

Fig. 10. Thermal diffusivities of materials in the coil, where stainless steel is indicated for reference and was not used in the coil.

the SMES applications. In fact, the performance values well exceeded the prototype design. Thus, the compact design of an actual system can be achieved and the running cost will be substantially reduced. Effective thermal design, arrangement of bundled Litz wires, leads to these high performances of the prototype. Further, the supporting structure with Dyneema FRP (DFRP) spacers in the coil winding enhances the thermal performance.

Since the coil consists of copper, aluminum, epoxy resign and DFRP, these materials have relatively high thermal diffusivities below $10 \mathrm{~K}$ as shown in Fig. 10. The time constants of thermal diffusivities for each component with the total amount of thickness used in the coil are estimated approximately $1 \mathrm{~s}$ around $4 \mathrm{~K}$ as shown in Fig. 11. Therefore, the heat generated within the coil was dissipated within the winding with a very short period.

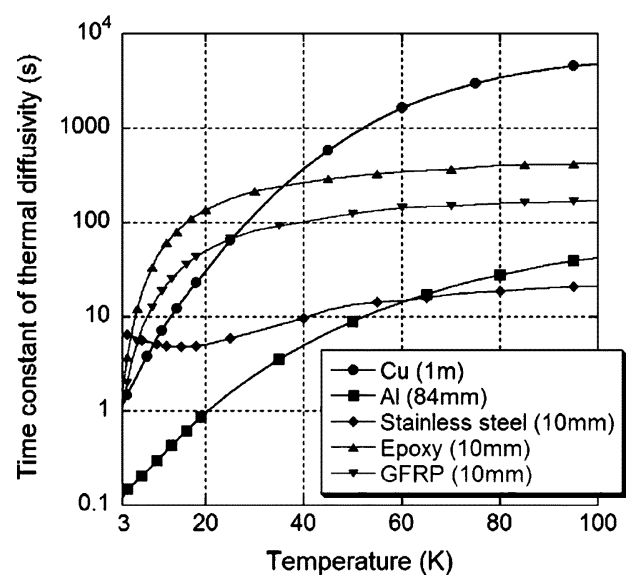

Fig. 11. Time constants of thermal diffusivities for each component with the total amount of thickness in the coil, where stainless steel $(10 \mathrm{~mm})$ is indicated for reference and was not used in the coil.

So, the spatial temperature distribution within the coil becomes negligibly small against heat generations. The detailed analysis including the heat transfer during the pulse operation and the comparison with the experimental data have been carried out in the related paper [6].

\section{SUMMARY}

We have successfully developed the conduction-cooled prototype LTS pulse-coil for UPS-SMES. The performance tests validate the design and fabrication technique of the coil. As a matter of fact, the data exceeded the design performance such as; the stored energy was $200 \mathrm{~kJ}$ instead of design value of $100 \mathrm{~kJ}$. Since the coil was designated for a pulse operation, the effective thermal diffusivity results in the rapid temperature stabilization. Consequently, the design philosophy has been established and the much compact system with a high efficiency can be developed.

\section{REFERENCES}

[1] M. Parizh et al., "Superconducting mangnetic energy storage for susstation applications," IEEE Trans. Appl. Supercond., vol. 7, no. 2, pp. 849-852, 1997.

[2] H. Salbert et al., "2 MJ SMES for an uninterruptible power supply," IEEE Trans. on Appl. Supercond., vol. 10, no. 1, pp. 777-779, 2000.

[3] S. Nagaya et al., "Development and performance results of 5 MVA SMES for bridging instantaneous voltage dips," IEEE Trans. on Appl. Supercond., vol. 14, no. 2, pp. 699-704, 2004.

[4] T. Mito et al., "Prototype development of a conduction-cooled LTS pulse coil for UPS-SMES," IEEE Trans. Appl. Supercond., vol. 15, no. 2, pp. 1935-1938, 2005.

[5] A. Kawagoe et al., "A new winding method to reduce AC losses in stable LTS pulse coils," IEEE Trans. Appl. Supercond., vol. 13, no. 2, pp. 2404-2407, 2003.

[6] — "Heat transfer properties of a conduction-cooled prototype LTS pulse coils for UPS-SMES," presented at the MT-19, THA09P006. 